\title{
Review of: "Intraoral scanners for capturing the palate and its relation to the dentition"
}

\author{
Alessandro Ricci
}

Potential competing interests: The author(s) declared that no potential competing interests exist.

The approach is interesting but the analysis process looks doubtful for the following reasons:

1. the oportunity of using the palatal rugae as a correct reference point doesn't look supported by the articles cited: written by the same autor of this very article, such articles report a small number of cases and give no clear indication about the oportunity to use rugae.

2. The results reported in the fugures 4 and 6 seem to give a regularity in the discrepancies among the CS scanners: i.e. patient n.2 got a high consistency no mather what's the reference point used, either palate or denture; patient n.10 looks to determine an error with the same pattern in both cases. This doesn't support the idea that scanners are more precise on smaller areas, even if this is confirmed from other studies.

The study analyzed a large and interesting amount of data but, for such discrepancies, some of the conclusions are not sure, in particular when it's said that "no systematic imprecision pattern was evident" 